\title{
The behavior of MFIE and EFIE at interior resonances and its impact in MOT late time stability
}

\author{
Lianrong Hong ${ }^{1,}$, Guiyu Tian ${ }^{1}$, Jinpeng Fang ${ }^{2}$, Gaobiao Xiao ${ }^{1}$ \\ ${ }^{1}$ Key Lab of Ministry of Education for Research of Design and EMC of High Speed Electronic System, Shanghai Jiao Tong University, \\ Shanghai, People's Republic of China \\ ${ }^{2}$ Shanghai Key Laboratory of Electromagnetic Environmental Effects for Aerospace Vehicle, Shanghai, China
}

Email address:

fannvwang@sjtu.edu.cn (L. Hong), redrivertgy@sjtu.edu.cn (G. Tian), gaobiaoxiao@sjtu.edu.cn(G. Xiao)

To cite this article:

Lianrong Hong, Guiyu Tian, Jinpeng Fang, Gaobiao Xiao. The Behavior of MFIE and EFIE at Interior Resonances and Its Impact in MOT Late Time Stability. American Journal of Electromagnetics and Applications. Vol. 1, No. 2, 2013, pp. 30-37.

doi: 10.11648/j.ajea.20130102.12

\begin{abstract}
In the vicinity of a certain interior frequency, the current density on the surface of a perfect electric conducting scatterer, when illuminated by an incident field, is divided into two parts: an induced surface current caused by the incident field and a resonance surface current associated with the interior resonance mode. Equivalent RLC circuit models are proposed respectively for PEC scatterers associated with the electric field integral equation (EFIE) and the magnetic field integral equation (MFIE). Using the circuit models, together with the power conservation law, the different behavior of the resonance surface currents associated with EFIE and MFIE is analyzed and checked with numerical examples in two-dimensional space. It is shown that the interior resonance behavior has significant influence on the late time stability associated with time domain EFIE and MFIE.
\end{abstract}

Keywords: MFIE, EFIE, Interior Resonance, MOT, Late Time Stability

\section{Introduction}

Interior resonance phenomena exist in solving electromagnetic scattering problems with surface integral equations (SIEs) [1]-[4]. Many authors have investigated the problem and have proposed detailed explanation on why interior resonance occurs and how to eliminate it [5]-[16]. Interior resonance problem exists only in the sense of numerical solutions since it does not exist in a real solid perfect electrically conducting (PEC) scatterer. Basically, interior resonance problem is due to the fact that boundary conditions are not strictly satisfied when using EFIE or MFIE alone. To use EFIE or MFIE alone may lead to nonzero fields within a solid PEC scatterer. As pointed out by D. Yaghjian [6], when applying EFIE or MFIE to the interior region of a scatterer, non-unique solutions are present at frequencies equal to the resonance frequencies of the interior cavity bounded by perfectly conducting surface because the Maxwell's equations allow homogeneous solutions; however, when applying EFIE or MFIE to the exterior region of the scatterer at these frequencies, homogeneous solutions are spurious solutions to Maxwell's equations because they do not satisfy all necessary boundary conditions. Augmented electric and magnetic field integral equations are proposed in [6] to eliminate the spurious solutions. A. F. Peterson discussed the nature of the resonance problem by checking the eigenvalue behavior of the EFIE and MFIE [15]. At frequencies equal to the resonance frequencies of the interior cavity, the eigenvalues of EFIE and MFIE vanish. Consequently, when the SIEs are solved numerically, the resultant coefficient matrices tend to be ill-conditioned. However, the numerical solutions, although incorrect, are usually stable and unique. The author in [15] considered that the stability of the solutions is mainly attributed to the fact that the discretization error associated with the matrix operator may prevent the eigenvalues at the interior resonance frequencies being as small as expected so that the coefficient matrices are in fact not singular. This explanation seems reasonable for EFIE, but not so convincing for MFIE, because numerical results show that in the case of EFIE, if finer mesh and higher precision are used, the condition number [17] of the coefficient matrix increases with no apparent upper bound; on the contrast, in the case of MFIE, the condition number of the coefficient matrix is always bounded. Further analysis in this paper 
shows that this limitation mainly comes from the power conservation law, which usually plays an important role in electromagnetic scattering problems [18].

Marching on in time (MOT) method is well adopted to solve time domain integral equations (TDIE). The late time instability of MOT-TDEFIE is still not a completely solved problem, which prevents its wide-spread application. It is known that interior resonance is perhaps the most dominant factor to cause MOT-TDEFIE instability [19]. On the contrary, TDMFIE is usually stable [20], although TDMFIE also suffers from interior resonance problem. In this paper, the behavior of the numerical solutions to EFIE and MFIE at the interior resonance frequencies is re-checked. It is found that the resonance current amplitude associated with EFIE is generally unbounded, while that associated with MFIE is bounded by incident field according to the power conservation law. This observation may help to explain the different stability property of TDEFIE and TDMFIE.

\section{Behavior near the Interior Resonance Frequencies}

Consider a PEC scatterer excited by an incident wave, as shown in Fig.1. The scatterer is enclosed by surface $S$. The exterior region $V$ is the problem region. According to equivalence principle, the interior area $V_{1}$ may be assumed to be any medium that is convenient for solving the original problem. For example, the inner side of surface $S$ (the side of $S$ facing $V_{1}$, hereafter denoted by $S^{-}$) can be assumed to be a PEC wall, or a perfect magnetically conducting (PMC) wall, or even an impedance wall. If the boundary condition at $S$ seen from region $V$ remains unchanged, these assumptions will not influence the solutions to the original problem in the exterior domain $V$.

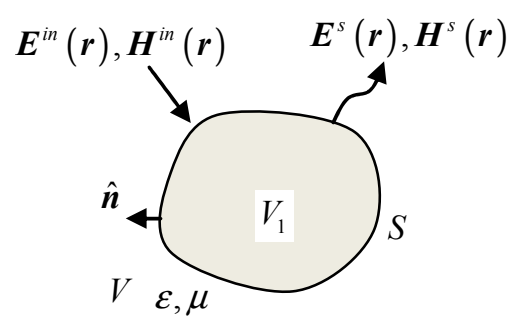

Figure 1. Scattering problem of a PEC scatterer.

When the PEC scatterer is illuminated by an incident wave, a surface current density $\boldsymbol{J}_{s}^{\text {ind }}$ will be induced on $S$, which must satisfy the following EFIE and MFIE simultaneously:

$$
\begin{gathered}
j \omega \mu \hat{\boldsymbol{n}} \times \mathcal{L}\left\{\boldsymbol{J}_{s}^{\text {ind }}\right\}=\hat{\boldsymbol{n}} \times \boldsymbol{E}^{\text {in }}(\boldsymbol{r}), \\
-\hat{\boldsymbol{n}} \times \mathcal{K}\left\{\boldsymbol{J}_{s}^{\text {ind }}\right\}+\frac{1}{2} \boldsymbol{J}_{s}^{\text {ind }}(\boldsymbol{r})=\hat{\boldsymbol{n}} \times \boldsymbol{H}^{\text {in }}(\boldsymbol{r}),
\end{gathered}
$$

The operators in (1) and (2) are defined in their usual way as follows

$$
\begin{aligned}
& \mathcal{K}\{\boldsymbol{f}\}=P . V . \oint_{S} \overline{\boldsymbol{G}}_{m}\left(\boldsymbol{r}, \boldsymbol{r}^{\prime}\right) \cdot \boldsymbol{f}\left(\boldsymbol{r}^{\prime}\right) d S^{\prime}, \\
& \mathcal{L}\{\boldsymbol{f}\}=P . V . \oint_{S} \overline{\boldsymbol{G}}_{e}\left(\boldsymbol{r}, \boldsymbol{r}^{\prime}\right) \cdot \boldsymbol{f}\left(\boldsymbol{r}^{\prime}\right) d S^{\prime},
\end{aligned}
$$

where $\overline{\boldsymbol{G}}_{e}\left(\boldsymbol{r}, \boldsymbol{r}^{\prime}\right)$ and $\overline{\boldsymbol{G}}_{m}\left(\boldsymbol{r}, \boldsymbol{r}^{\prime}\right)$ are the electric and magnetic dyadic Green's functions in region $V$, respectively. The residual term stemmed from the singularity of the Green's function is separated from the integral in (2). The notation P.V. means Cauchy principal value.

Equations (1) and (2) come from the fact that the electric field and magnetic field in the interior region of the PEC scatterer are zeroes. Evidently, the tangential components of the electric field and the magnetic field on $S^{-}$are all zeroes, therefore, the surface $S^{-}$acts not only like a PEC wall but also like a PMC wall. The induced current density on $S$ is uniquely determined for a specified incident wave, and disappears with the incident wave. However, MFIE or EFIE is usually applied alone in electromagnetic scattering problems. As pointed out in [6], nontrivial solutions to the homogeneous equations associated with EFIE and MFIE may exist at frequencies equal to the resonance frequencies of the interior cavity bounded by a perfectly conducting surface $S^{-}$or a perfectly magnetic conducting surface $S^{-}$. In these situations, the surface current at $S$ contains two parts: one part is the induced current $\boldsymbol{J}_{s}^{\text {ind }}$ depending on the incident wave; the other part is related to nontrivial solutions to the associated homogeneous equation. We may denote

$$
\boldsymbol{J}_{s}(\boldsymbol{r})=\boldsymbol{J}_{s}^{\text {ind }}(\boldsymbol{r})+\boldsymbol{J}_{s n}^{e}
$$

for EFIE, and

$$
\boldsymbol{J}_{s}(\boldsymbol{r})=\boldsymbol{J}_{s}^{\text {ind }}(\boldsymbol{r})+\boldsymbol{J}_{s n}^{h}
$$

for MFIE. The vectors $\boldsymbol{J}_{s n}^{e}$ and $\boldsymbol{J}_{s n}^{h}$ are respectively the nontrivial solutions to the homogeneous equations associated with EFIE and MFIE, which are termed as eigencurrents in [15]. Subscript ' $s n$ ' is used because nontrivial solutions usually exist at discrete frequency points.

The spatial distribution property of the nontrivial solutions $\boldsymbol{J}_{s n}^{e}$ and $\boldsymbol{J}_{s n}^{h}$ is independent of incident fields. Hence, these currents can be considered as independent current sources. However, the behavior of $\boldsymbol{J}_{s n}^{e}$ and $\boldsymbol{J}_{s n}^{h}$ is quite different from each other.

The nontrivial solutions $\boldsymbol{J}_{s n}^{e}$ associated with EFIE satisfy

$$
j \omega \mu \hat{\boldsymbol{n}} \times \mathcal{L}\left\{\boldsymbol{J}_{s n}^{e}\right\}=0
$$


If interpreted explicitly, (5) tells that at frequencies corresponding to the eigenvalues of (5), there exist nonzero surface current sources on $S$, the tangential components of whose radiated electric fields on $S$ are zeroes.

Although $\boldsymbol{J}_{s n}^{e}$ can be seen as independent current sources, they cannot radiate waves to the exterior region $V$ [6] [14]. This feature can be proved easily using Huygens' equivalence principle. On the other hand, the surface currents at frequencies equal to the resonance frequencies of the interior cavity bounded by a perfectly conducting surface $S$ are also subject to equation (5). Therefore, nontrivial solutions $\boldsymbol{J}_{s n}^{e}$ actually correspond to currents associated with interior resonance modes of the virtual cavity bounded by a PEC surface $S^{-}$. Therefore, $\boldsymbol{J}_{s n}^{e}$ are usually referred to as nontrivial solutions or interior resonance currents. Because the tangential component of the electric field by the non-zero $\boldsymbol{J}_{s n}^{e}$ on $S^{-}$is zero, the surface $S^{-}$can be regarded as a PEC wall. Intuitively speaking, $\boldsymbol{J}_{s n}^{e}$ are current sources placed infinitely near a closed PEC surface, hence, it is reasonable to predict that $\boldsymbol{J}_{s n}^{e}$ can not radiate fields to the exterior region $V$.

The nontrivial solutions $\boldsymbol{J}_{s n}^{h}$ to the homogeneous equation associated to MFIE satisfy,

$$
-\hat{\boldsymbol{n}} \times \mathcal{K}\left\{\boldsymbol{J}_{s n}^{h}\right\}+\frac{1}{2} \boldsymbol{J}_{s n}^{h}(\boldsymbol{r})=0, \quad \boldsymbol{r} \in S,
$$

Since $\boldsymbol{J}_{s n}^{h}$ exist under no excitation, they can also be treated as independent sources just like $\boldsymbol{J}_{s n}^{e}$. Intuitively, $\boldsymbol{J}_{s n}^{h}$ are current sources placed infinitely near a closed PMC surface, so it can radiate fields to the exterior region $V$. It has also been verified that at resonance modes of a cavity formed by a perfect magnetically conducting surface $S^{-}$, the associated resonance magnetic current on the cavity wall satisfies exactly the same equation of (6). Therefore, nontrivial solutions to equation (6) are corresponding to the resonance modes of the interior cavity bounded by a PMC wall.

It is important to notice that solenoidal static currents also satisfy (5), but do not satisfy (6). This means that EFIE admits interior resonance at $\omega=0$, or DC resonance, while MFIE has no interior resonance at DC.

Theoretically, the interior resonance occurs only at discrete frequency points, and the spectrum should be discrete single lines. However, in practical applications, the SIEs need to be solved with numerical methods. The spectrum may be blurred due to discretization errors, and the interior resonance might occur in a narrow band in the vicinity of the discrete resonance frequencies.

Equivalent circuit mode can be used to illustrate this problem. In the case of EFIE, at interior resonance frequencies, the tangential components of the total electric fields should vanish in theory, so there should be no ohmic loss on the PEC surface $S$. The interior cavity associated with the scatterer in this situation can be modeled as an ideal resonator. However, due to the discretization errors, the numerically calculated tangential components of the total electric fields may have nonzero small values, which will lead to a numerical ohmic loss $P_{\text {loss }}$ on the surface $S$

$$
P_{l o s s}=\oint_{S}\left[\boldsymbol{E}^{i n}(\boldsymbol{r})-j \omega \mu \mathcal{L}\left\{\boldsymbol{J}_{s}^{i n d}+\boldsymbol{J}_{s n}^{e}\right\}\right] \cdot \boldsymbol{J}_{s n}^{e^{*}}(\boldsymbol{r}) d S
$$

where the upper script “*” means conjugate. This numerical loss makes the interior cavity a dumping resonator. Assume that $P_{\text {loss }}$ is dissipated by an effective resistor $R_{\text {num }}^{e}$, then the behavior of the interior resonator may be approximately described using the equivalent $R L C$ circuit model shown in Fig.2(a). The Q-factor of the cavity resonator will not be infinitely large because of the effect of $R_{n u m}^{e}$. Therefore, the corresponding resonance current is finite, and the interior resonance may span to occupy a narrow spectrum band. If finer mesh structure is adopted to get higher numerical accuracy, $R_{\text {num }}^{e}$ tends to become smaller, leading to larger Q-factor, narrower band and larger resonance current. This feature is in agreement with the observation in other papers (e.g., [15]).

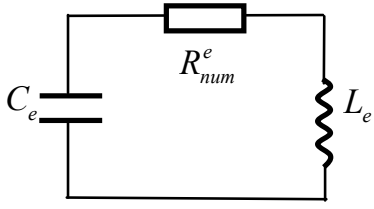

(a)

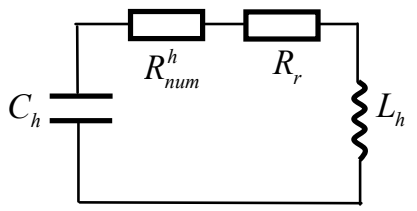

(b)
Figure 2. (a) Circuit model for interior resonance associated with EFIE. (b) Circuit model for interior resonance associated with MFIE.

In the case of MFIE, the resonance current may radiate fields to the exterior region. This can be regarded as a radiation loss seen from the interior cavity. Therefore, the interior resonance associated with MFIE can be described using the equivalent $R L C$ circuit model in Fig.2 (b), with $R_{\text {num }}^{h}$ accounting for the numerical loss and $R_{r}$ accounting for the radiation loss. Because $R_{r}$ is usually much larger than $R_{\text {num }}^{h}$, the Q-factor associated with the interior cavity in the case of MFIE is much smaller than that in EFIE. Consequently, comparing to the case of EFIE, the spectrum band associated with MFIE at a resonance frequency is much wider and the amplitude of the resonance current is much smaller.

Although the spatial distribution of the resonance currents is independent on the incident fields, their excitation is strongly dependent on the incident fields. Consider the scattered fields by an incident field at a certain interior resonance frequency. As has been pointed out previously, the total surface current contains an induced current and a resonance current. In the case of EFIE, we can write 


$$
\hat{\boldsymbol{n}} \times\left[\boldsymbol{E}^{i n}(\boldsymbol{r})-j \omega \mu \mathcal{L}\left\{\boldsymbol{J}_{s}^{\text {ind }}+\boldsymbol{J}_{s n}^{e}\right\}\right]=0, \quad \boldsymbol{r} \in S .
$$

When solving this equation with method of moment, the condition number of the coefficient matrix tends to be very large at interior frequencies, as can also be predicted using the $R L C$ circuit mode shown in Fig.2 (a). The solution may be unstable if iteration solver is used. However, since equation (8) is a Fredholm type equation of the second kind, a stable and unique solution can still be found using pseudo inversion technique [7]. Theoretically, the scattered fields are not influenced by the resonance current in the case of EFIE. The induced current can be separated from the total current by

$$
\hat{\boldsymbol{n}} \times\left[\boldsymbol{H}^{i n}(\boldsymbol{r})+\boldsymbol{H}^{s}\left(\boldsymbol{J}_{s}^{\text {ind }}+\boldsymbol{J}_{s n}^{e}\right)\right]=\boldsymbol{J}_{s}^{\text {ind }}, \quad \boldsymbol{r} \in S .
$$

Power conservation must be observed in electromagnetic scattering problems. Because $\boldsymbol{J}_{s n}^{e}$ is independent to the incident fields, and do not radiate waves into the exterior region $V$, the scattered fields are totally caused by $\boldsymbol{J}_{s}^{\text {ind }}$. In the case of a PEC scatterer, the power conservation law requires that

$$
\oint_{S_{1}}\left[\boldsymbol{E}^{\text {in }}(\boldsymbol{r})+\boldsymbol{E}^{s}\left(\boldsymbol{J}_{s}^{\text {ind }}\right)\right] \times\left[\boldsymbol{H}^{\text {in }}(\boldsymbol{r})+\boldsymbol{H}^{s}\left(\boldsymbol{J}_{s}^{\text {ind }}\right)\right]^{*} \cdot \hat{\boldsymbol{n}} d S=0,(10)
$$

where $S_{1}$ is an arbitrary closed surface enclosing the scatterer. Since the numerical ohmic loss is usually negligible, the relation of (10) is always easy to observe. The power conservation relation poses no limitation to the resonance current $\boldsymbol{J}_{s n}^{e}$, the amplitudes of which may possibly become very large if the discretization error is very small.

In the case of MFIE, the total surface current $\left(\boldsymbol{J}_{s}^{\text {ind }}+\boldsymbol{J}_{s n}^{h}\right)$ satisfy

$$
-\hat{\boldsymbol{n}} \times \mathcal{K}\left\{\boldsymbol{J}_{s}^{i n d}+\boldsymbol{J}_{s n}^{h}\right\}+\frac{1}{2}\left(\boldsymbol{J}_{s}^{i n d}+\boldsymbol{J}_{s n}^{h}\right)=\hat{\boldsymbol{n}} \times \boldsymbol{H}^{i n}(\boldsymbol{r}), \quad \boldsymbol{r} \in S
$$

The spatial distribution of $\boldsymbol{J}_{s n}^{h}$ is also independent to the incident fields. However, since $\boldsymbol{J}_{s n}^{h}$ can radiate waves to the exterior region $V$, the amplitudes of $\boldsymbol{J}_{s n}^{h}$ are limited by the incident fields according to the power conservation law. For example, if the scatterer is a perfect conductor, the total outgoing power must be equal to the total ingoing power. Because of this limitation, when solving (11) with MoM, the condition number of the coefficient matrix is always a finite value, and the amplitude of the resonance current is bounded. This feature has also been predicted using the $R L C$ circuit model illustrated in Fig. 2 (b).

Two-dimensional examples are used to verify the above observations. A PEC cylinder is illuminated by a TM plane wave with $\boldsymbol{E}=\hat{\boldsymbol{a}}_{z} e^{-j k x}[\mathrm{~V} / \mathrm{m}]$. The permittivity and permeability of the background medium are $\varepsilon, \mu$, respectively. Let $\boldsymbol{J}_{s n}^{T M, e}=\hat{\boldsymbol{z}} j_{s n}^{T M, e}$ denote the surface current density of the $n$th interior resonance mode associated with EFIE when it is excited by the TM plane wave. Then $\boldsymbol{J}_{s n}^{T M, e}$ satisfy

$$
j \omega \mu \hat{\boldsymbol{\rho}} \times \oint_{C} \frac{1}{4 j} H_{0}^{(2)}\left(k\left|\boldsymbol{\rho}-\boldsymbol{\rho}^{\prime}\right|\right) \boldsymbol{J}_{s n}^{T M, e}\left(l^{\prime}\right) d l^{\prime}=0,
$$

where $H_{0}^{(2)}\left(k\left|\boldsymbol{\rho}-\boldsymbol{\rho}^{\prime}\right|\right)$ is the zeroth-order Hankel function of the second kind. Expanding the Hankel function using the addition formula, and exchanging the operation of summation and integration yields

$$
\hat{\boldsymbol{\varphi}} \sum_{n=-\infty}^{\infty} J_{n}(k a) H_{n}^{(2)}(k a) \tilde{j}_{s n}^{T M, e} e^{j n \phi}=0,
$$

where $\tilde{j}_{s n}^{T M, e}$ is the Fourier transform of $j_{s n}^{T M, e}(\phi)$.It can be seen from (12) that nonzero currents may exist only under the condition of $J_{n}(k a)=0$, namely, $k_{n l}^{T M, e} a=v_{n l}$, with $v_{n l}$ being the $l$ th root of the $n$th order Bessel function. The discrete interior resonance frequencies $f_{n l}^{T M, e}$ can be determined accordingly. Since $v_{-n l}=v_{n l}$, it is not difficult to verify that $f_{-n l}^{T M, e}=f_{n l}^{T M, e}$. Denote the normalized resonance surface current of the $n$th mode as

$$
\boldsymbol{j}_{s n}^{T M, e}(\phi)=\hat{\boldsymbol{z}} e^{j n \phi}
$$

In the vicinity of $f_{n l}^{T M, e}$, the total surface current can be expressed as $\left(\boldsymbol{J}_{s}^{\text {ind }}+C_{n}^{e} \boldsymbol{j}_{s n}^{T M, e}\right)$, where $C_{n}^{e}$ is a coefficient depending on the discretization error and the frequency discrepancy from the resonance frequency.

Numerical experiments are carried out in the vicinity of $k a=v_{01}$, sweeping from $k a L=2.4048255574357$ to $k a H=2.4048255574757$ with incremental step of $\Delta k a=1.0 \times 10^{-12}$. The circumference of the cylinder is divided into 900 equal-length segments, and 900 roof-top basis functions are used to expand the surface current densities and the rotated tangential field components. Standard Galerkin's scheme is used to discretize the corresponding EFIE. The calculated total surface currents are almost exactly of the form of $\left(\boldsymbol{J}_{s}^{\text {ind }}+C_{n}^{e} \boldsymbol{j}_{s n}^{T M, e}\right)$. Fig.3 (a) shows the condition number of the coefficient matrix, and Fig.3 (b) shows the magnitude of $2 C_{n}^{e}$. Both have a peak at $k a 0$. In the above-described numerical setup, the amplitude of the resonance current at the peak is calculated to be $2 C_{n}^{e}=2.079[\mathrm{~A} / \mathrm{m}]$, which is about 371 times of that of the induced surface current. Fig.3(c) shows that the numerical value of the induced current density agree very well with that obtained by using mode expansion method. The bi-static radar cross sections (RCSs) at three frequency points are plotted in Fig.3 (d), where the current density 
and the RCSs are all normalized with wavelength. It can be seen that although very large resonance currents are mixed in the total currents, the calculated RCSs are almost not polluted.

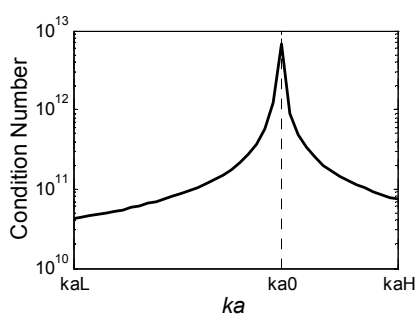

(a)

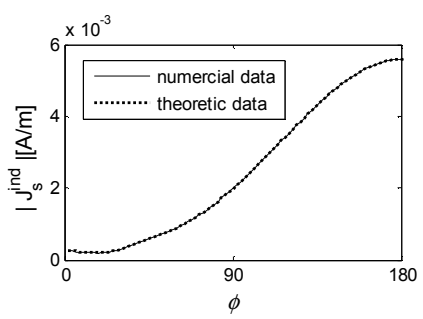

(c)

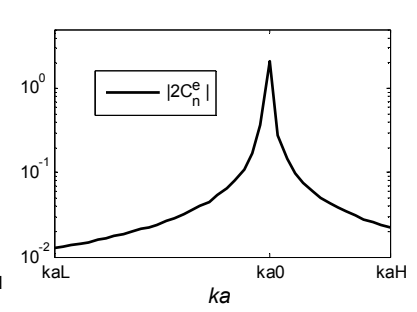

(b)

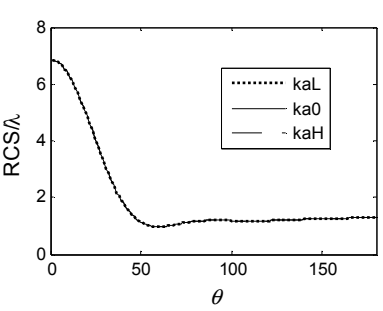

(d)
Figure 3. The numerical results in the vicinity of $k a=v_{01}$. (a) The condition number of the coefficient matrix. (b) The amplitudes of the resonance surface currents. (c) The induced surface current at ka0 . (d) The bi-static RCSs at three frequency points

Let $\boldsymbol{J}_{s n}^{T M, h}=\hat{\boldsymbol{z}} j_{s n}^{T M, h}$ denote the surface current density of the $n$th interior resonance mode associated with the MFIE on the surface of the PEC cylinder under the excitation described previously. Then $\boldsymbol{J}_{s n}^{T M, h}$ satisfy

$$
-\hat{\boldsymbol{\rho}} \times \oint_{C} \nabla\left[\frac{1}{4 j} H_{0}^{(2)}\left(k\left|\boldsymbol{\rho}-\boldsymbol{\rho}^{\prime}\right|\right)\right] \times\left[\hat{\boldsymbol{z}} J_{s n}^{T M, h}\left(\phi^{\prime}\right)\right] d l^{\prime}+\hat{\boldsymbol{z}} J_{s n}^{T M, h}=0
$$

which can be transformed into

$$
\hat{\boldsymbol{z}} \sum_{n=-\infty}^{\infty} J_{n}^{\prime}(k a)\left[J_{n}(k a)-j Y_{n}(k a)\right] \tilde{J}_{s n}^{T M, h} e^{j n \phi}=0,
$$

where $\tilde{j}_{s n}^{T M, h}$ is the Fourier transform of $j_{s n}^{T M, h}(\phi)$. Nonzero surface currents may exist only under the condition of $J_{n}^{\prime}(k a)=0$, i.e., $k_{n l}^{T M, h} a=\mu_{n l}$, with $\mu_{n l}$ being the $l$ th root of the derivative function of the $n$th order Bessel function. The corresponding normalized resonance surface currents also have the form of $\boldsymbol{j}_{s n}^{T M, h}(\phi)=\hat{\boldsymbol{z}} e^{j n \phi}$, and the total surface currents can be expressed as $\left(\boldsymbol{J}_{s}^{\text {ind }}+C_{n}^{h} \boldsymbol{j}_{s n}^{T M, h}\right)$, where $C_{n}^{h}$ is the coefficient of the surface current associated with the $n$th resonance mode. As has been discussed previously, $C_{n}^{h}$ is bounded by the power conservation relation. In order to check this relation closely, the incident electric and magnetic fields are separated into an ingoing part and an outgoing part, respectively. When a PEC cylinder is present, the outgoing wave will include not only the outgoing part of the incident wave, but also the scattered fields by the currents $\left(\boldsymbol{J}_{s}^{i n d}+C_{n}^{h} \boldsymbol{j}_{s n}^{T M, h}\right)$, which can be derived to be

$$
\begin{gathered}
E_{z}^{s}\left(J_{s}^{i n d}\right)=\sum_{-\infty}^{\infty} a_{n} H_{n}^{(2)}(k \rho) e^{j n \phi-j n \pi / 2}, \\
H_{\phi}^{s}\left(J_{s}^{i n d}\right)=-\frac{k}{j \omega \mu} \sum_{-\infty}^{\infty} a_{n} H_{n}^{\prime(2)}(k \rho) e^{j n \phi-j n \pi / 2},
\end{gathered}
$$

and

$$
\begin{gathered}
E_{z n}^{T M, h}\left(J_{s n}^{T M, h}\right)=C_{n}^{h} b_{n} J_{n}(k a) H_{n}^{(2)}(k \rho) e^{j n \phi-j n \pi / 2}, \\
H_{z n}^{T M, h}\left(J_{s n}^{T M, h}\right)=-\frac{k}{j \omega \mu} C_{n}^{h} b_{n} J_{n}(k a) H_{n}^{\prime(2)}(k \rho) e^{j n \phi-j n \pi / 2},(1)
\end{gathered}
$$

where $a_{n}=-E_{0} J_{n}(k a) / H_{n}^{(2)}(k a), b_{n}=-\frac{\pi a \omega \mu}{2} e^{j n \pi / 2}$.

Equations (17) and (18) are derived by directly solving the scattered fields of the resonance surface currents using the Green's function and the addition formula. Taking advantage of the orthogonality of functions $e^{j n \phi}$, one can see that the power conservation relation must be observed for every harmonic component. The ingoing power of the $n$th harmonic component is found to be

$$
P_{n}^{i n}=2 \pi \rho\left\langle S_{n}\right\rangle^{i n}=\frac{E_{0}^{2}}{2 \omega \mu},
$$

while the total outgoing power can be obtained as follows,

$$
P_{n}^{\text {out }}=2 \pi \rho\left\langle S_{n}\right\rangle^{\text {out }}=\frac{E_{0}^{2}}{2 \omega \mu}\left|1-C_{n}^{h}\left[\frac{2 H_{n}^{(2)}(k a)}{E_{0} H_{n}^{(1)}(k a)} b_{n} J_{n}(k a)\right]\right|^{2}
$$

Power conservation law requires that $P_{n}^{\text {in }}=P_{n}^{\text {out }}$, i.e.,

$$
\mid 1-C_{n}^{h}\left[\frac{2 H_{n}^{(2)}(k a)}{E_{0} H_{n}^{(1)}(k a)} b_{n} J_{n}(k a)\right]=1 .
$$

Equation (21) lays a clear constraint to the amplitude of the resonance surface currents associated with MFIE. The maximum resonance surface current occurs when the second term in the left hand side of (21) equals to 2, which leads to

$$
C_{n, \max }^{h}=E_{0} H_{n}^{(1)}(k a)\left[H_{n}^{(2)}(k a) b_{n} J_{n}(k a)\right]^{-1}
$$

It can be further proven that $C_{-n, \text { max }}^{h}=C_{n, \text { max }}^{h}$. The overall resonance surface current that has the largest amplitude is found to be 


$$
J_{s n, \text { max }}^{h}=C_{n, \text { max }}^{h} e^{j n \phi}+C_{-n, \text { max }}^{h} e^{-j n \phi}=2 C_{n, \text { max }}^{h} \cos (n \phi) .
$$

As a numerical example, the behavior of the interior resonance mode in the vicinity of $k a=\mu_{11}=1.841$ is numerically analyzed. The variable $k a$ changes from $k a L=1.841202$ to $k a H=1.841282$, with an incremental step of $\Delta k a=0.000002$. The numerical setup is the same as that in the example for EFIE. The resonance surface current associated with this mode is $2 C_{1}^{h} \cos (\phi)$. The condition number of the coefficient matrix is shown in Fig. 4(a), and the calculated amplitude of the resonance surface current is plotted in Fig.4 (b). Both reach their maximum value at $k a 0$. The amplitude of the resonance surface current is smaller than the amplitude of the induced current in this case. The coefficient $2 C_{1, \max }^{h}$ at $k a 0$ is calculated to be $(0.00193+j 0.00247)$. The theoretic value of $2 C_{1, \max }^{h}$ at $k a 0$ can be obtained using (22). The discrepancy of the two results is less than $0.1 \%$. The calculated surface currents at $k a 0$ are plotted in Fig.4(c), and the RCSs associated with them are plotted in Fig.4(d). Apparently, the interior resonance current has significant influence on the far fields.

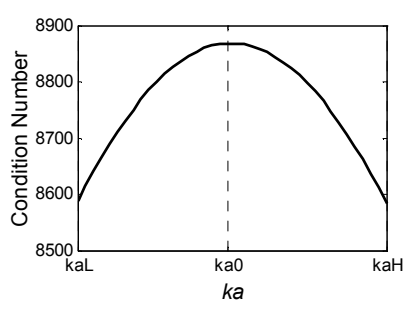

(a)

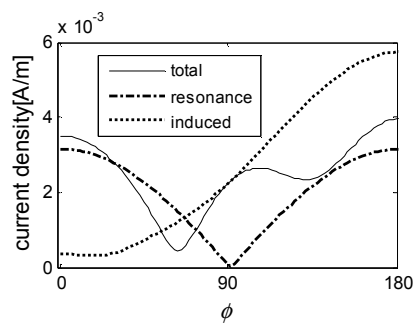

(c)

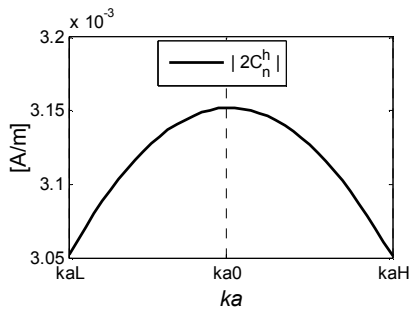

(b)

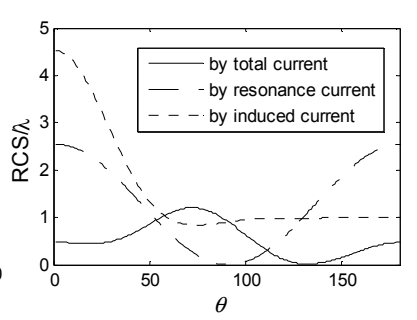

(d)
Figure 4. The numerical results in the vicinity of $k a=\mu_{11}$. (a) The condition number of the coefficient matrix. (b)The amplitudes of the resonance surface currents. (c) The calculated surface currents at ka0 (d) The corresponding bi-static RCSs.

\section{Impact on the MOT Late Time Instability}

Two points worth to be emphasized concerning the behavior of EFIE and MFIE at interior resonance frequencies: (1) EFIE admits DC resonance, while MFIE does not; (2) the amplitudes of the resonance currents associated with EFIE are almost unbounded, while those associated with MFIE are bounded by incident fields. In TDIE, the incident fields usually consist of wide band frequency components. Therefore, many interior resonances can be excited. However, according to the orthogonality property of interior resonance fields, only the part of the incident fields in a narrow band near an interior resonance frequency can contribute to the excitation of that interior resonance. In other words, the effective excitation field for each interior resonance is very small.

In TDEFIE, the amplitudes of interior resonance currents, including DC resonance current, may possibly grow very large even for small excitations. However, in MFIE, the amplitudes of interior resonance currents are always very small because they are strictly limited by the small effective incident fields.

In TDEFIE and TDMFIE, the influence of discretization error is also different. Since higher accuracy generally leads to larger resonance current in EFIE, one cannot expect to lower the interior resonance currents by increasing numerical accuracy, even if it is difficult to conclude that higher accuracy inevitably cause larger interior resonance currents in TDEFIE. However, in TDMFIE, the effective excitation frequency band tends to become narrower with higher numerical accuracy, and the effective excitation field for each interior resonance tends to become smaller. Consequently, the interior resonance current amplitudes tend to decease with increasing numerical accuracy.

We use the following remark to conclude this section: the interior resonances have significant impact on the late time stability of MOT-TDEFIE solvers, but have very limited influence on the late time stability of MOT-TDMFIE solvers. This is in consistent with the observation by other researchers that TDMFIE is usually stable [20].

In the following, simple numerical results are provided to verify this remark.

A PEC sphere with radius $1 \mathrm{~m}$ is assumed to be illuminated by a Gaussian-shaped pulse plane wave expressed by

$$
\overrightarrow{\boldsymbol{E}}=\hat{\boldsymbol{a}}_{x} \frac{1}{\sqrt{\pi}} \exp \left[-(v t-6-\hat{\boldsymbol{z}} \cdot \overrightarrow{\boldsymbol{r}})^{2}\right](\mathrm{V} / \mathrm{m}) .
$$

The surface currents on the sphere are calculated using MOT-TDEFIE and MOT-TDMFIE, respectively. The sphere is meshed to 47 triangles, resulting 114 RWGs. We intentionally adopted a time consuming strategy for calculating the mutual coupling coefficients between RWGs in order to get high accuracy: the inner surface integration is performed analytically while the outer surface integration is performed using 25 points Gaussian quadrature for every pair of RWGs. We try to exclude the influence of numerical error on the late time instability in 
this way, at the cost of long CPU time. Implicit MOT scheme is used to solve the original TDEFIE and TDMFIE, not their differentiated versions. The time step is set to be $\Delta t=0.5 \mathrm{~ns}$, corresponding to a space-time discretization ratio of $\chi=c \Delta t / l_{\max }=0.18$, where $l_{\max }$ is the largest edge length in the mesh triangles. The sphere center is located at the origin. The surface current near the point $(0,0,-1 \mathrm{~m})$ is shown in Fig.5. It can be seen from Fig. 5(a) that the early time results agree well except that the result calculated by using TDEFIE has larger ripples. Fig. 5(b) shows the wide scope results till fifty-thousand time steps ( $25 \mu \mathrm{s}$ ). The TD-MFIE result is stable, however, the TDEFIE result is divergent.

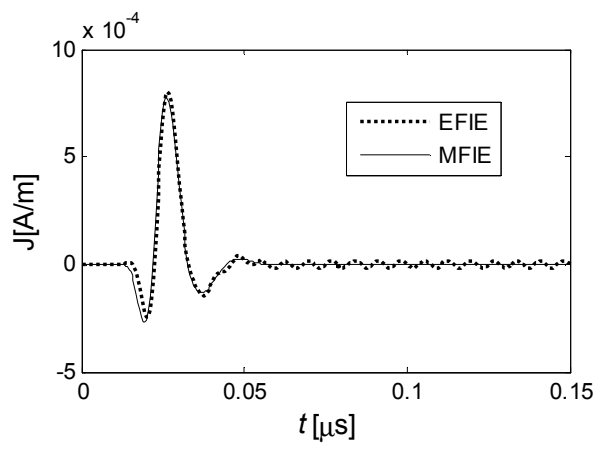

(a)

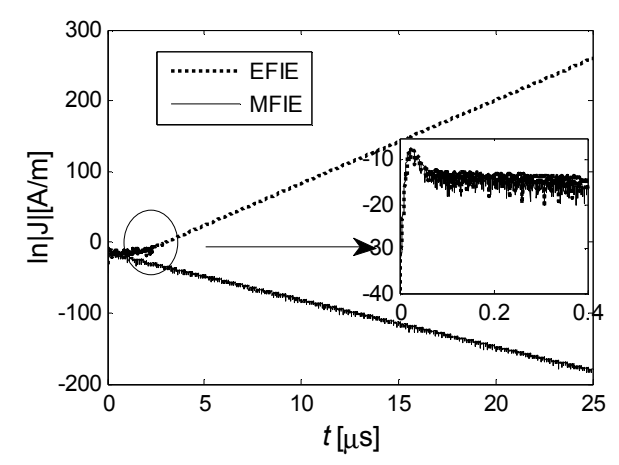

(b)

Figure 5. PEC sphere. (a) Early time results. (b) Late time stability property.

The second scatterer examined is a PEC cubic with side length of $1 \mathrm{~m}$. Its mesh structure contains 154 triangles and 231 RWGs. The same strategy is adopted for calculating the coupling coefficients. The incident field is assumed to be a modulated Gaussian-shaped plane wave, with center frequency of $100 \mathrm{MHz}$, bandwidth of $50 \mathrm{MHz}$, and time delay of $c t_{p}=24$. The space-time discretization ratio is chosen to be $\chi=0.35$. The surface current density near the point of $(-0.2 \mathrm{~m}, 0.1 \mathrm{~m},-0.5 \mathrm{~m})$ is shown in Fig.6. Similar to the previous example, the TDMFIE result is definitely stable. However, the TDEFIE result seems stable till thirty-thousand time steps $(15 \mu \mathrm{s})$, but it eventually diverges, although very slowly.

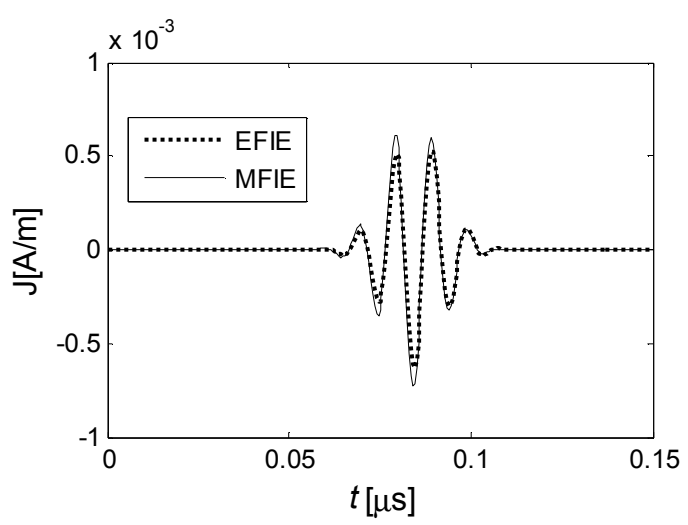

(a)

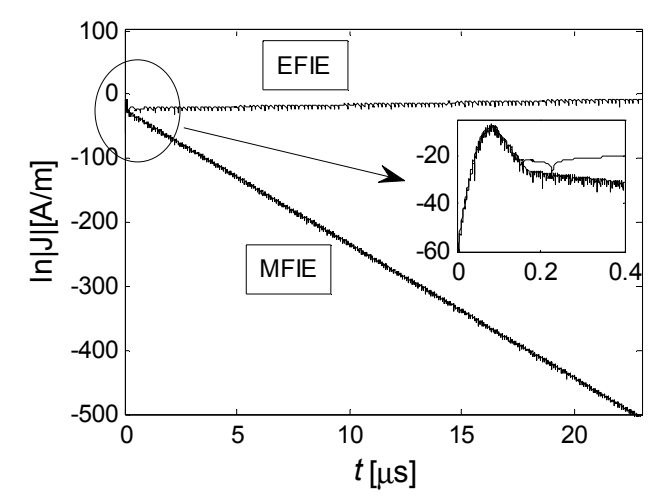

(b)

Figure 6. PEC cubic. (a) Early time results.(b) Late time stability property.

\section{Conclusions}

In this paper, circuit models are used to explain the difference in behaviors of the interior resonances associated with EFIE and MFIE. The power conservation relation is checked in these situations. The results show that in the case of EFIE, the amplitudes of the resonance currents are essentially unbounded. Small excitation may possibly cause very large resonance currents; while in the case of MFIE, the amplitudes of the resonance currents are all strictly bounded by the incident fields according to power conservation law. It is observed with numerical examples in this paper that the interior resonance currents have significant impact on the late time instability of MOT-TDEFIE method, but will not deteriorate the stability of MOT-TDMFIE method.

\section{Acknowledgment}

This work was supported by SAST Fundation 2013. 


\section{References}

[1] A. J. Burton, and G. F. Miller, "The application of integral equation methods to the numerical solution of some exterior boundary value problems," Proc. Roy. Soc., Lond. A, vol. 323, pp. 201-210, 1971.

[2] N. Morita, "Resonant solutions involved in the integral equation approach to scattering from conducting and dielectric cylinders," IEEE Trans. Antennas Propagat., vol. 27, no.6, pp. 869-871, Nov. 1979.

[3] A. A. Mohsen, A. R. A. Helaly and M. H. Fahmy, "The corrected induced surface current for arbitrary conducting objects at resonance frequencies," IEEE Trans Antennas Propagat., vol. 43, no. 5, pp. 448-452, May 1995.

[4] K. F. A. Hussein, "Effect of internal resonance on the radar cross section and shield effectiveness of open spherical enclosures," Progress In Electromagnetics Research, PIER 70, pp. 225-246, 2007.

[5] Mautz, J. R., and Harrington R. F., "A combined-source solution for radiation and scattering from a perfectly conducting body," IEEE Trans. Antennas Propagat., Vol. 27, No. 4, pp. 445-454, April 1979.

[6] A. D. Yaghjian, "Augmented electric- and magnetic-field integral equations, " Radio Sci., vol. 16, no. 6, pp. 987-1001, Nov. 1981.

[7] K. T. Sarkar and M. S. Rao, "A simple technique for solving $E$-field integral equations for conducting bodies at internal resonances," IEEE Trans Antennas Propagat., vol. 30, no. 6, pp. 1250-1254, Nov. 1982.

[8] F. X. Canning, "Protecting EFIE-based scattering computations from effects of interior resonances," IEEE Trans Antennas Propagat., vol. 39, no. 11, pp. 1545-1552, Nov. 1991.

[9] J. C. Monzon, and N. J. Damaskos, "A scheme for eliminating internal resonances: the parasitic body technique," IEEE Trans. Antennas Propagat., vol. 42, no. 8, pp. 1089-1091, Aug. 1994.

[10] F. Andriulli and E. Michielssen, "A regularized combined field integral equation for scattering from 2-D perfect electrically conducting objects, "IEEE Trans. Antennas Propagat., vol. 55, no. 9, pp. 2522-2529, Sept. 2007.

[11] J. Lee and S. Nam, "Protecting the method of auxiliary sources (MAS) solutions from the interior resonance problem," IEEE Microw. and Wireless Comp. Lett., vol. 15, no. 3, pp. 186-188, Mar. 2005.

[12] R. J. Adams and N. J. Champagne, "A numerical implementation of a modified form of the electric field integral equations," IEEE Trans. Antennas Propagat., vol. 52, no. 9, pp. 2262-2266, Sept. 2004.

[13] S. H. Christiansen and J. C. Nedelec, "A Preconditioner for the Electric Field Integral Equation Based on Calderon Formulas," SIAM J. Numer. Anal., vol. 40, no. 3, pp. 1100-1135, 2002.

[14] W. C. Chew and J. M. Song, "Gedanken experiments to understand the internal resonance problems of electromagnetic scattering," Electromagnetics, vol. 27, no. 8, pp. 457-471, Nov. 2007.

[15] A. F. Peterson, "The interior resonance problem associated with surface integral equations of electromagnetics: Numerical consequences and a survey of remedies," Electromagn., vol. 10, no. 3, pp. 293-312, 1990.

[16] Özgür Ergül, and Levent Gürel, "Efficient solution of the electric-field integral equation using the iterative LSQR algorithm," IEEE Antennas Wireless Propagat. Let., vol. 7, pp. 36-39, 2008.

[17] C. A. Klein, and R. Mittra, "An application of the "condition number' concept to the solution of scattering problems in the presence of the interior resonant frequencies," IEEE Trans. Antennas Propagat., vol. 23, no. 3, pp. 431-435, May 1975.

[18] R. E. Collin, "Rayleigh Scattering and Power Conservation," IEEE Trans. Antennas Propagat., vol. 29, no.5, pp. 795-798, Sept. 1981.

[19] F. P. Andriulli, K. Cools, F. Olyslager, and Eric Michielssen, "Time domain Calderón Identities and their application to the integral equation analysis of scattering by PEC objects part II: stability," IEEE Trans. Antennas Propagat., vol. 57, no. 8, pp. 2365-2375, 2009.

[20] B. Shanker, A. A. Ergin, K. Aygun, and E. Michielssen, "Analysis of transient electromagnetic scattering from closed surfaces using a combined field integral equation," IEEE Trans. Antennas Propagat., vol. 48, no. 7, pp. 1064-1074, 2000. 